Review

\title{
Short-term and long-term plasticity at corticostriatal synapses: Implications for learning and memory
}

\author{
Massimiliano Di Filippo a,b ${ }^{\mathrm{a}}$, Barbara Picconi ${ }^{\mathrm{b}}$, Michela Tantucci ${ }^{\mathrm{a}, \mathrm{b}}$, Veronica Ghiglieri ${ }^{\mathrm{b}}$, \\ Vincenza Bagetta ${ }^{\mathrm{b}, \mathrm{c}}$, Carmelo Sgobio $^{\mathrm{b}}$, Alessandro Tozzi ${ }^{\mathrm{a}, \mathrm{b}}$, Lucilla Parnetti ${ }^{\mathrm{a}}$, Paolo Calabresi ${ }^{\mathrm{a}, \mathrm{b}, *}$ \\ a Clinica Neurologica, Laboratori di Neurologia Sperimentale, Universita' di Perugia, Perugia, Italy \\ ${ }^{\mathrm{b}}$ I.R.C.C.S. Fondazione Santa Lucia, Rome, Italy \\ ' Università della Calabria, Dipartimento Farmaco-biologico, Arcavata di Rende, Cosenza, Italy
}

\section{A R T I C L E I N F O}

\section{Article history:}

Received 30 April 2008

Received in revised form

10 September 2008

Accepted 15 September 2008

Available online 2 October 2008

\section{Keywords:}

Synaptic plasticity

Long-term potentiation

Long-term depression

Striatum

Basal ganglia

Memory

\begin{abstract}
A B S T R A C T
The striatum is the major division of the basal ganglia, representing the input station of the circuit and arguably the principal site within the basal ganglia where information processing occurs.

Striatal activity is critically involved in motor control and learning. Many parts of the striatum are involved in reward processing and in various forms of learning and memory, such as reward-association learning. Moreover, the striatum appears to be a brain center for habit formation and is likely to be involved in advanced stages of addiction.

The critical role played by the striatum in learning and cognitive processes is thought to be based on changes in neuronal activity when specific behavioral tasks are being learned.

Accordingly, excitatory corticostriatal synapses onto both striatal projecting spiny neurons and interneurons are able to undergo the main forms of synaptic plasticity, including long-term potentiation, long-term depression, short-term forms of intrinsic plasticity and spike timing-dependent plasticity.

These specific forms of neuroplasticity allow the short-term and long-term selection and differential amplification of cortical neural signals modulating the processes of motor and behavioral selection within the basal ganglia neural circuit.
\end{abstract}

(C) 2008 Elsevier B.V. All rights reserved.

\section{Contents}

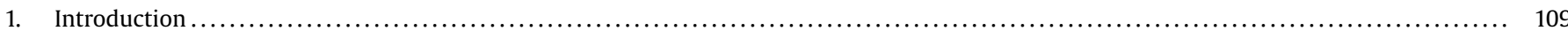

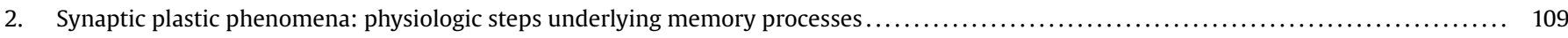

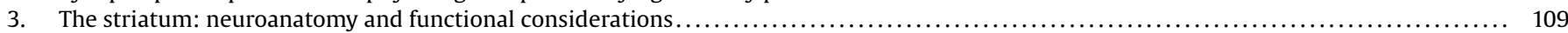

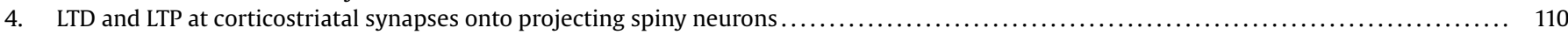

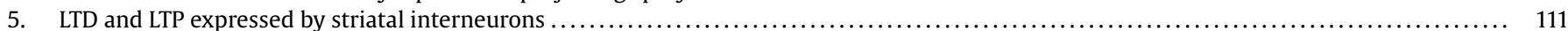

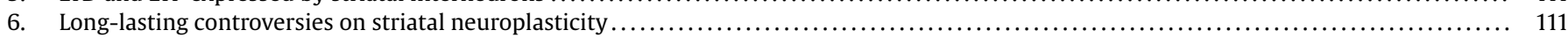

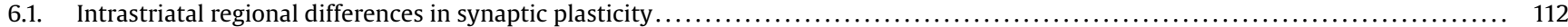

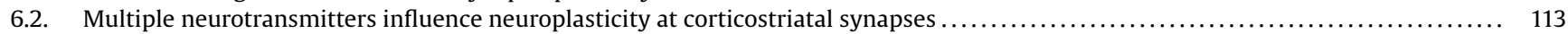

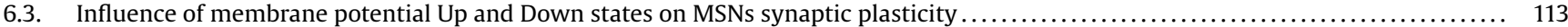

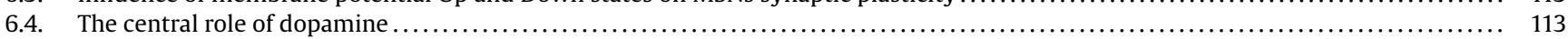

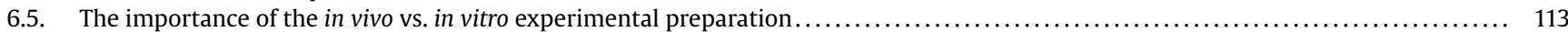

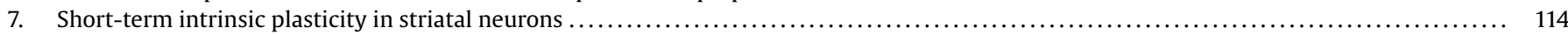

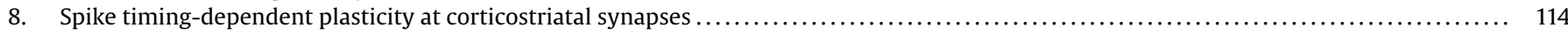

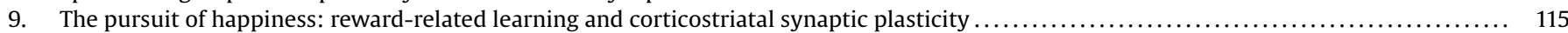

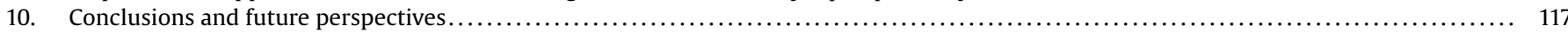

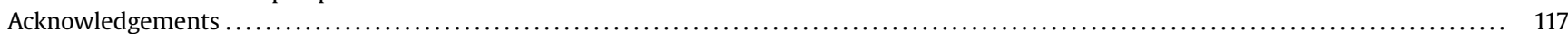

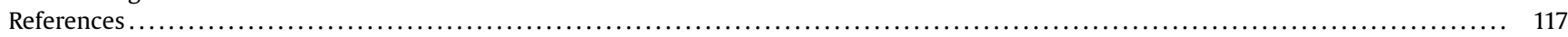

\footnotetext{
* Corresponding author at: Clinica Neurologica, Facoltà di Medicina e Chirurgia, Università degli Studi di Perugia, Ospedale S. Maria della Misericordia, 06156 Perugia, Italy. Tel.: +39 0755784230 ; fax: +39 0755784229.

E-mail address: calabre@unipg.it (P. Calabresi).
} 


\section{Introduction}

The nucleus striatum, the input station of the basal ganglia neural circuit, receives excitatory input from different regions of the cerebral cortex and a prominent input directly from thalamic nuclei and is thought to be a primary substrate for numerous forms of learning and memory and for controlling behavioral output.

The excitatory drive from corticostriatal glutamatergic afferents activates striatal neurons which, in turn, alter the activity of neurons throughout the entire basal ganglia circuitry.

The connectivity within the striatum has begun to be unravelled, but the integrative significance of the intrastriatal networks is still far from being completely elucidated.

Projecting GABAergic spiny neurons dominate in striatum (95\%), and exert their physiological functions by closely interacting with several subtypes of interneurons including three subtypes of GABAergic neurons and large aspiny cholinergic interneurons [38].

In addition to having a general role in motor learning [56] and in the initiation of a wide variety of behaviors [60], the striatum appears to be a brain center for habit formation and is likely to be involved in the advanced stages of addiction, when drug use progresses toward a compulsive behavior mostly directed at drugseeking [30].

The critical role played by the striatum in learning, cognition, behavioral control and its pathological implications are likely to be based on changes in neuronal activity when specific behavioral tasks are being learned.

Excitatory corticostriatal synapses onto both striatal projecting spiny neurons and interneurons are able to undergo the main forms of synaptic plasticity [18].

In particular, both long-term depression (LTD) and long-term potentiation (LTP) have been demonstrated to occur at these synapses and are known to be accompanied by a number of other events able to influence synaptic weights and to prevent neuronal network destabilization.

All these specific forms of neuroplasticity allow the short-term and long-term selection and differential amplification of cortical neural signals modulating the processes of motor and behavioral selection within the basal ganglia neural circuit.

\section{Synaptic plastic phenomena: physiologic steps underlying memory processes}

One of the most fascinating and enigmatic properties of the brain is represented by its plastic potential. Indeed, experiences are able to modulate neural activities resulting in long-lasting modifications of neural circuits, subsequently influencing thoughts, feelings and behaviors [22].

Since the classic descriptions of nervous system structure and function by Ramon y Cajal and Sherrington, the capacity of the brain to translate transient experiences into persistent memory traces has been attributed to the occurrence of long-lasting, activitydependent changes in the efficacy of synaptic communication [48].

The idea that the plastic properties of synapses might support activity-dependent changes in synaptic efficiency and thus represent the "engram" of memory was further refined in the late 1940s by Donald Hebb, who proposed a coincidence-detection rule in which the synapse linking two neurons is strengthened if the two cells are active at the same time [1].

Nevertheless, experimental support for these hypotheses was lacking until the early 1970s, when it was shown for the first time that the repetitive activation of hippocampal excitatory synapses resulted in a long-lasting increase in synaptic strength, named LTP [48].
Activity-dependent synaptic potentiation occurs within milliseconds, can persist for hours or days and is expressed as a persistent increase in the size of the synaptic component of the evoked response, recorded either from individual neurons or from a larger cells population [22]. The question of whether the modification in synaptic efficiency occurring during activitydependent plasticity is due primarily to postsynaptic modification in glutamate AMPA receptors or to a presynaptic change in neurotransmitter release is still matter of debate [48]. Nevertheless, evidence suggest that probably both presynaptic and postsynaptic mechanisms play a role, and that probably neurons of different neuronal networks can vary in terms of the specific forms of synaptic plasticity they express.

Together with LTP, LTD remains the other main accepted vertebrate model underlying learning and memory.

It is still debated whether LTD plays a specific, independent role in memory processes or if it serves as an adjunct to LTP, for example by enhancing signal-to-noise ratio, renormalizing synaptic weights and/or deleting previously stored information [49].

A third, potentially important form of homosynaptic plasticity is "depotentiation", which results from the reversal of an established LTP by a low-frequency stimulation protocol (LFS, $1-5 \mathrm{~Hz}$ ) and seems to be distinct from de novo LTD [52,55].

Depotentiation and other regulatory processes, such as synaptic scaling, are as important as Hebbian LTP and LTD in determining the consequences of synaptic plasticity on neural network functioning [1].

Indeed, Hebbian forms of plasticity, acting independently at each synapse gain power but also acquire stability problems, potentially destabilizing postsynaptic firing rates, reducing them to zero or increasing them excessively [1].

\section{The striatum: neuroanatomy and functional considerations}

The striatum is the major division of the basal ganglia. The corticostriatal axons, arising from the whole cortical mantle, mainly innervate the striatal medium-sized projecting spiny neurons (MSNs), which account for the large majority of striatal neurons and which, in turn, project either directly to the output nuclei of the basal ganglia (in the so-called "direct pathway") or to the external segment of the globus pallidus (GPe) (in the so called "indirect pathway") and thence to the output nuclei [32] (Fig. 1).

MSNs are inhibitory neurons that use the neurotransmitter $\gamma$ aminobutyric acid (GABA) and interact to some degree through local GABAergic collaterals. Activity through the 'direct pathway' from the striatum to the output basal ganglia nuclei (substantia nigra pars reticulata and the internal globus pallidus, SNr and GPi) provides powerful inhibitory control of these basal ganglia output neurons. By contrast, activity through the parallel 'indirect pathway' leads to increased activity of excitatory glutamatergic neurons in the subthalamic nucleus (STN), which induces strong excitation of the SNr and GP [32].

A thinly regulated balance of output nuclei activity through the direct and the indirect pathways is thought to be essential for normal function of the basal ganglia. Dopamine (DA) arising from the substantia nigra pars compacta (SNpc) participates to information processing within the striatum, as well as acetylcholine $(\mathrm{ACh})$, GABA and other neurotransmitters (such as nitric oxide) released by striatal interneurons.

In vivo intracellular recordings of spontaneous activity of neostriatal spiny cells have revealed a two-state behavior of this neuronal population [69]. Indeed, MSNs show characteristic shifts of membrane potential between two preferred levels, one more 


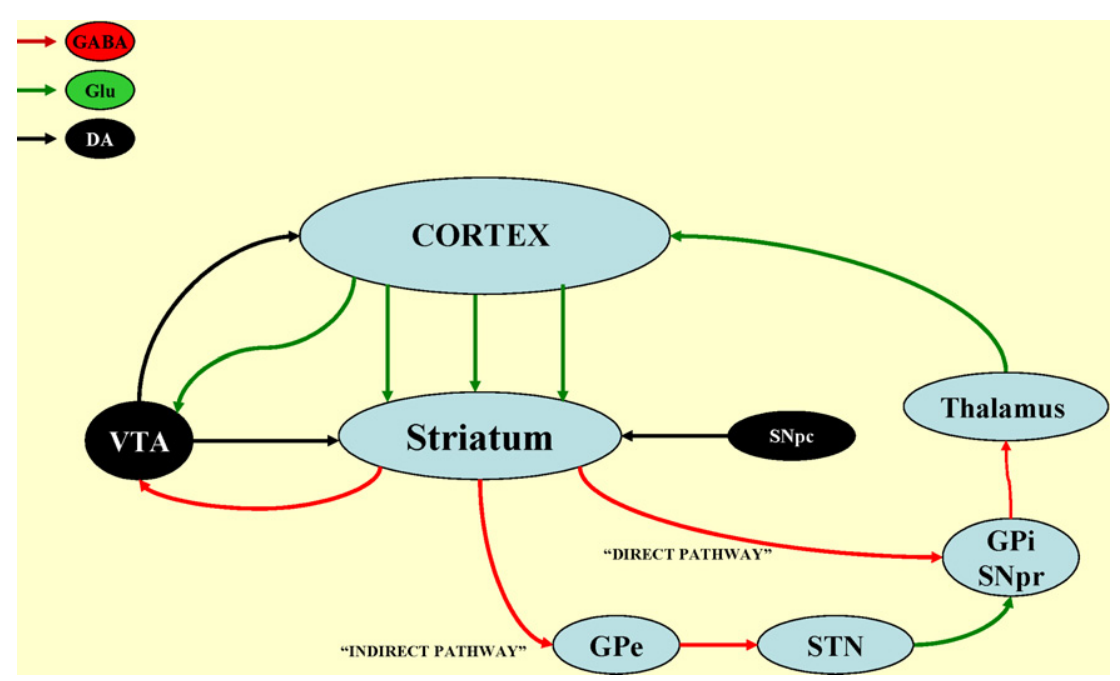

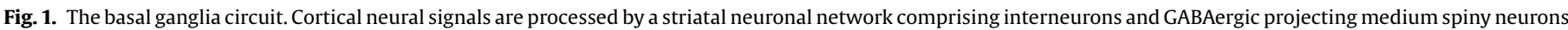

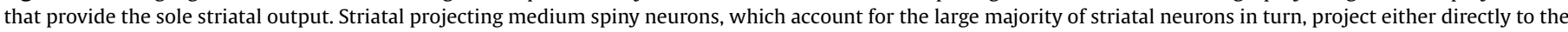

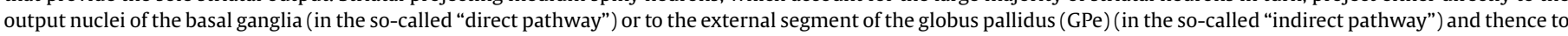

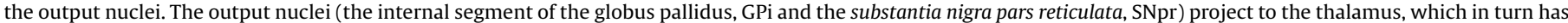

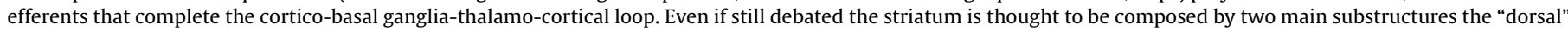

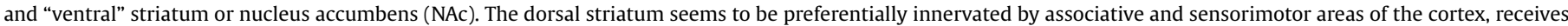

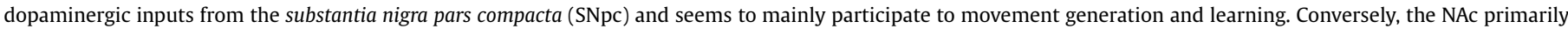

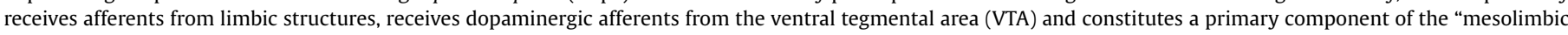

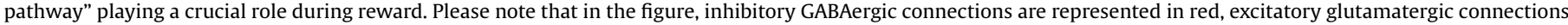
are in green and dopaminergic connections in black. Abbreviations: DA, Dopamine; GABA, gamma-aminobutyric acid; Glu, glutamate; STN, subthalamic nucleus.

polarized, called the Down state (varying from -61 to $-94 \mathrm{mV}$ ), and one more depolarized level, called the Up state (varying from -71 to $-40 \mathrm{mV}$ ) triggered by an increased activity of many convergent corticostriatal neurons (and possibly thalamostriatal neurons as well) [69]. During the Up state, additional excitatory inputs or an alteration in the balance of excitatory and inhibitory inputs might lead to the firing of action potentials. This phasic activity of the MSNs leads to altered rates and patterns of firing in the output nuclei and, as specified below, deeply influences the induction of the main forms of synaptic plasticity at corticostriatal synapses.

A physiological synaptic transmission and plasticity in the striatum is thought to be essential for motor control and learning [56]. Indeed, when a subpopulation of striatal neurons is activated, it provides strong inhibition to a subpopulation of pallidal neurons (which have a high resting activity) and thus indirectly removes the tonic inhibition from a particular target motor center, thereby activating its motor program [32].

Accordingly, a reduced DAergic innervation to the striatum, due to SNpc degeneration during Parkinson's disease (PD) leads to the alteration of MSNs activity [9], to the loss of the main forms of synaptic plasticity $[18,55]$ and to the onset of severe motor symptoms, such as hypokinesia and difficulty in initiating different motor patterns $[42,43]$.

Striatal activity does not seem to be only involved in motor control, but it is also thought to be critical in controlling behavioral output [60]. Many parts of the striatum are involved in reward processing and in various forms of learning and memory, such as habit learning, goal-directed-instrumental and reward-association learning and procedural and emotional learning [35,60,72].

The involvement of the striatum in numerous forms of learning is likely to be based on changes in neuronal activity occurring when specific behavioral and motor tasks are being learned. The basal ganglia indeed, and in particular the striatum, are capable of selection and differential amplification of neural signals, in the short-term through lateral inhibition and modification of MSNs membrane properties and in the long-term by long-term forms of neuroplasticity, which can preserve or alter the process of motor and behavioral selection [4].

Almost all the forms of Hebbian plasticity have been demonstrated at excitatory corticostriatal synapses onto striatal projecting neurons and some of them seem also to be expressed by striatal interneurons [18]. Together with the main forms of Hebbian plasticity (LTP and LTD) corticostriatal synapses are able to undergo non-Hebbian forms of neuroplasticity, short-term forms of intrinsic plasticity, spike timing-dependent plasticity, depotentiation and other essential mechanisms preventing neural network destabilization [4].

\section{LTD and LTP at corticostriatal synapses onto projecting spiny neurons}

LTD and LTP at corticostriatal synapses were first reported on 1992 [7,8] when pioneering studies of long-term activitydependent modifications at glutamatergic corticostriatal synapses demonstrated that the high-frequency stimulation (HFS) of corticostriatal fibres using three trains of pulses at $100 \mathrm{~Hz}$, in association with postsynaptic neuronal firing, was able to induce a LTD of corticostriatal transmission onto striatal projecting MSNs [7]. In particular, it was demonstrated that tetanic stimulation produced a LTD $(>2 \mathrm{~h})$ of both extracellularly recorded field potentials and intracellularly recorded excitatory postsynaptic potentials (EPSPs) and that subthreshold tetanic stimulation, which under control condition did not cause LTD, induced LTD when associated with membrane depolarization.

This work also investigated the basis of the pharmacological manipulation of this form of synaptic plasticity providing insights into the physiological role of striatal neurotransmitters in the regulation of corticostriatal neuroplasticity. 
Bath application of an NMDA receptors antagonist did not affect the expression of LTD as well as the $G_{A B A}$ receptor antagonist bicuculline. Conversely, striatal LTD was significantly reduced by the pretreatment of the slices with an antagonist of glutamate metabotropic receptors [7].

The critical role of DA in the modulation of striatal neuroplasticity has been evident since this first description of LTD. Indeed, both D1 and D2 DA receptors antagonists were able to block LTD induction which was also absent in slices obtained from rats in which the nigrostriatal DA system was lesioned by unilateral nigral injection of 6-hydroxydopamine.

Thus, it was evident that the expression of a LTD of corticostriatal synaptic transmission required: (i) membrane depolarization and action potential discharge of the postsynaptic cell during the conditioning tetanus, (ii) activation of glutamate metabotropic receptors, and (iii) the coactivation of D1 and D2 DA receptors [7].

Subsequently, it has been demonstrated that the generation of striatal LTD requires a $\mathrm{Ca}^{2+}$ influx through voltage-dependent nifedipine-sensitive $\mathrm{Ca}^{2+}$ channels, a sufficient intracellular free $\mathrm{Ca}^{2+}$ concentration and the activation of $\mathrm{Ca}^{2+}$-dependent protein kinases [10]. Subsequent studies also investigated which glutamate metabotropic receptor subtype is selectively involved in LTD induction at corticostriatal synapses showing that the expression of this form of synaptic plasticity seems to require the selective activation of mGluR1 [33,34].

Differently from LTD, LTP was initially found to be expressed by corticostriatal synapses after the removal of $\mathrm{Mg}^{2+}$ ions from the extracellular medium, an experimental condition able to deinactivate NMDA glutamate receptors [8].

Accordingly, corticostriatal LTP was found to be dependent on the activation of these receptors [8]. As for striatal LTD, also striatal LTP was demonstrated to be critically dependent on DA [19]. In particular, a role of D1/D5 DA receptors has been repeatedly demonstrated [39,58].

Notably, both striatal LTD and LTP are influenced not only by the activation of DA receptors by DA arising from the SNpc but also by several other neurotransmitters (Fig. 2).

With regard to this latter point, a critical role has been suggested to be played by striatal cholinergic interneurons which represent the main source of striatal ACh [57].

Unlike most striatal neurons, cholinergic interneurons are autonomous pacemakers. This autonomous activity of cholinergic interneurons has led to them being referred to as 'tonically active neurons' (TANs) in behaving animals [57].

Striatal ACh bidirectionally interacts with DA either in modulating striatal neurotransmission or in influencing synaptic plasticity [17] and this cross talk seems to be critically important because of its behavioral significance [23].

In particular, ACh seems to influence directly (that is, not through the modulation of DA release) both LTP and LTD induction at corticostriatal synapses. Activation of M1-like muscarinic receptors is required for the induction of corticostriatal LTP [13] probably via a PKC (protein kinase C)-mediated mechanism, while a reduced concentration of synaptic ACh seems to facilitate LTD induction by lowering the M1 muscarinic receptor tone and thus disinhibiting Cav1.3 $\mathrm{Ca}^{2+}$ channels [66].

A role has also been suggested to be played by M2 muscarinic receptors. Indeed, M2-like muscarinic receptors antagonists enhance striatal corticostriatal LTP [12], suggesting that the activation of M2-like muscarinic receptors may exert a negative influence on striatal LTP, probably by reducing the release of glutamate from corticostriatal fibres [12].

The spectrum of neurotransmitters exerting a potential influence on striatal neuroplasticity is huge and is still expanding.
A specific interest is now directed toward the understanding of the specific role played by endogenous endocannabinoids in the modulation of striatal LTD.

Indeed, in contrast to classical neurotransmitters, endogenous cannabinoids can act as retrograde synaptic messengers, being released from postsynaptic neurons and traveling backward across synapses, finally suppressing neurotransmitter release by the activation of presynaptic CB1 receptors [70].

In particular in the striatum it has been demonstrated that a form of LTD is dependent on the activation of presynaptic CB1 receptor by postsynaptically released endocannabinoids acting as retrograde messengers [29].

More in particular, in 2007 Kreitzer and Malenka have demonstrated, by utilizing BAC transgenic mice that only indirect pathway-MSNs seem to express this form of endocannabinoiddependent LTD [41].

The evidence of a form of LTD mediated by the postsynaptic release of endocannabinoids should not lead to the underestimation of the role of DA in modulating striatal synaptic plasticity. Indeed, DA D2 receptors activation has been linked to endocannabinoid release in the striatum [31] and critically modulates endocannabinoids mediated LTD [40].

\section{LTD and LTP expressed by striatal interneurons}

As introduced above, albeit studies of the cellular organization of the neostriatum have mainly focused on projecting MSNs, a specific and critical role in the regulation of the neostriatal circuitry is played by a population of interneurons, including three types of GABAergic cells (one coexpressing parvalbumin, one calretinin and one nitric oxide synthase) and cholinergic interneurons [38,57].

It is worth to remember that also striatal interneurons, as projecting MSNs, are able to undergo long-lasting modifications of the strength of synaptic transmission.

In particular, striatal cholinergic interneurons express a form of LTP after the tetanic stimulation of cortico/thalamostriatal fibres that has been demonstrated to require a rise in intracellular $\mathrm{Ca}^{2+}$ concentration and DA D5, but not DA D2 or NMDA receptors activation $[16,63]$.

The ability to express synaptic plastic changes has been also investigated in striatal GABAergic interneurons and, more specifically, in the interneuronal subtype known as fast-spiking interneuron, because of its peculiar electrophysiological characteristics [6].

These cells have been demonstrated to express either LTP or LTD, depending on the pattern of synaptic stimulation, and to require NMDA receptors activation to undergo these forms of synaptic plasticity [28].

\section{Long-lasting controversies on striatal neuroplasticity}

As described above, the first evidence that corticostriatal synapses were able to undergo activity-dependent changes in the efficacy of synaptic transmission has been provided since the beginning of the 1990s with the initial description of an NMDAindependent corticostriatal LTD [7,8].

Conversely, in vitro LTP was initially observed only when HFS was coupled with the pharmacological manipulation of the slice medium, e.g. LTP was elicited by HFS when $\mathrm{Mg}^{2+}$ was omitted from the extracellular solution [8], allowing the deinactivation of NMDA receptors, or when HFS was coupled with a pulsatile application of extracellular $\mathrm{K}^{+}$and DA [68].

According to these first observations the main hypotheses were that (i) LTD was the normal, "physiological" form of plasticity at corticostriatal glutamatergic synapses and that (ii) the 


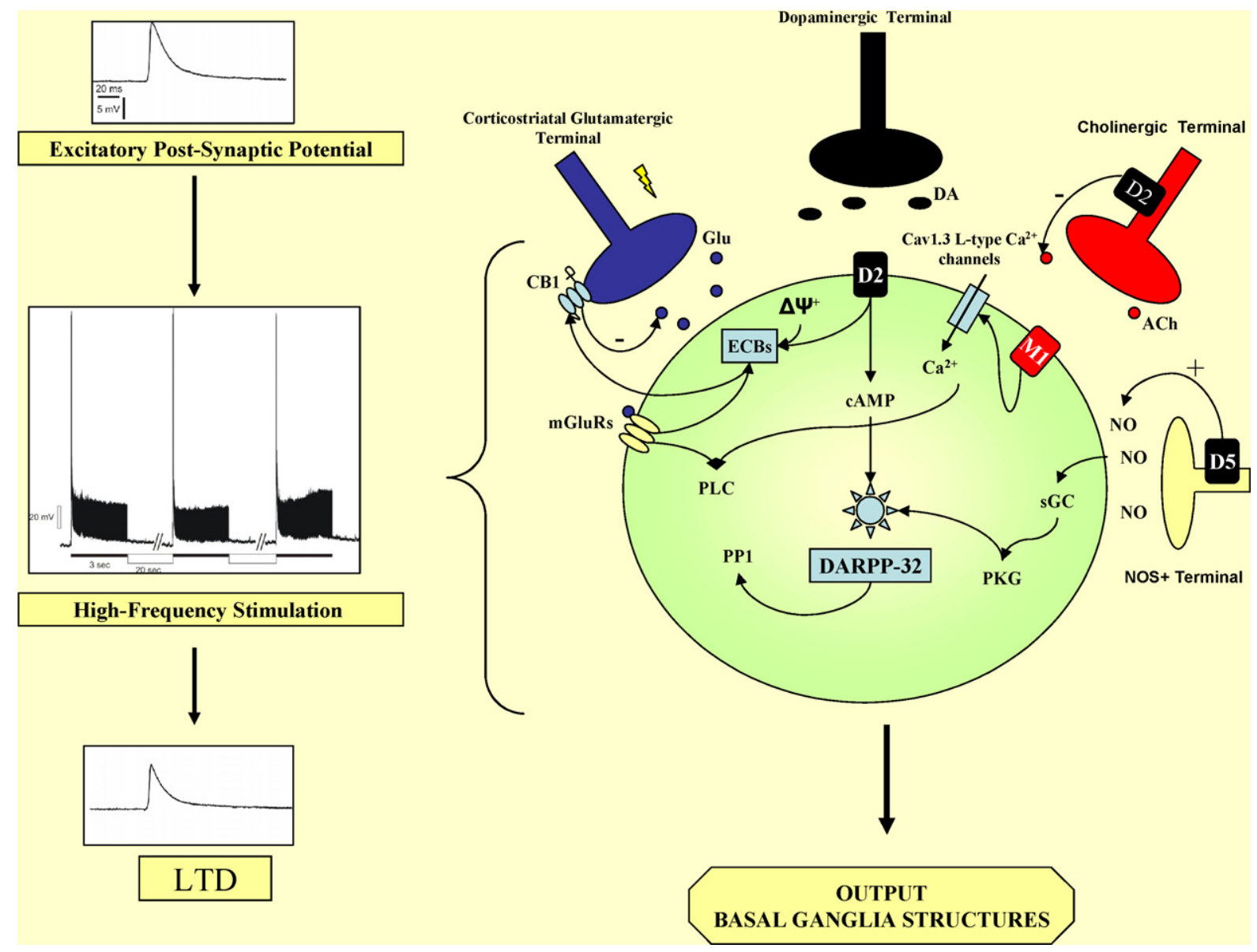

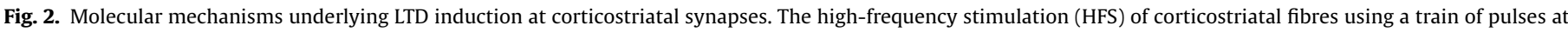

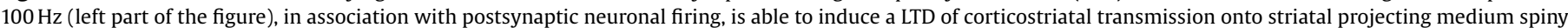

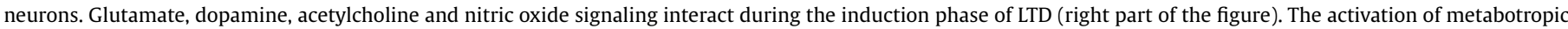

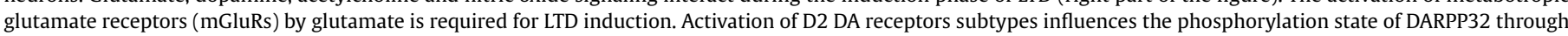

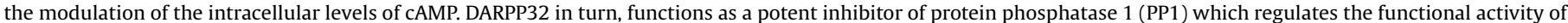

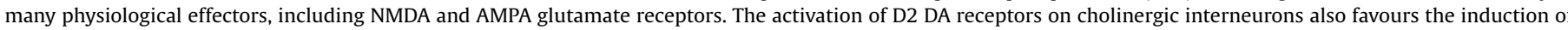

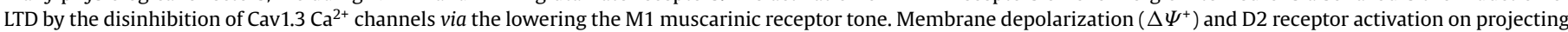

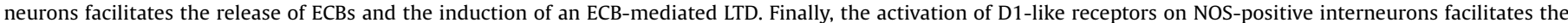

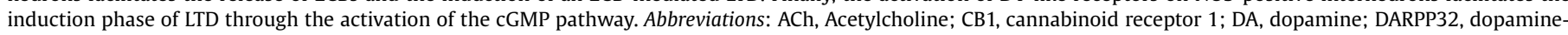

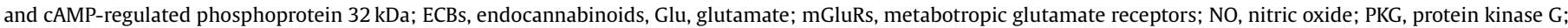
PLC, phospholipase C; PP1, protein phosphatase 1; sGC, soluble guanylyl cyclase.

switch from LTD to LTP involved the activation of NMDA receptors.

Subsequent either in vitro or in vivo experimental efforts have partially challenged this view, demonstrating that the regulation of striatal neuroplasticity is more complex and profoundly affected by several conditions, including experimental procedures.

In particular, several factors have been demonstrated to profoundly affect the final effect of a repetitive transmission at corticostriatal synapses, such as (i) the striatal region taken into account, (ii) the developmental age, (iii) the influence of several neurotransmitter systems, (iv) the physiological state of MSNs, (v) the degree of DAergic stimulation, and (vi) the in vivo vs. in vitro experimental conditions.

\subsection{Intrastriatal regional differences in synaptic plasticity}

As far as it concerns the regional differences observed within the striatum in terms of neuroplasticity, it is worth noting that distinct rules of synaptic plasticity seem to mirror an anatomical, functional and behaviorally relevant division of the striatum [67].
Indeed, even if still debated, it is widely accepted that the striatum consists of a dorsal sensorimotor part and a ventral portion processing limbic information that, although very similar with respect to neural cytoarchitecture and neurotransmitter content, seem to vary with regard to afferent and efferent circuitry and receiving DAergic projections.

To date, various ways of subdividing the striatum have been suggested and the traditional idea of a dorsal "sensorimotor" part and a ventral, "limbic" portion has been challenged and partially substituted by a theory supporting a more graded, dorsolateral-toventromedial functional organization [67].

This sub-division of the striatum seems to be reflected by the existence of distinct rules of synaptic plasticity and to influence, together with developmental age, the final effect of the high-frequency activation of excitatory striatal synapses [53]. Accordingly, in 2000, it has been reported that corticostriatal synapses in the dorsolateral region of the anterior striatum tend to switch from predominant LTP to predominant LTD depending on developmental age while synapses in the dorsomedial anterior striatum exhibit a propensity to express an NMDA-receptor 
dependent form of LTP across the entire developmental time period examined [53].

\subsection{Multiple neurotransmitters influence neuroplasticity at corticostriatal synapses}

A complex neurochemical balance is probably responsible for the "direction" (i.e. LTD vs LTP) of the synaptic modification following the HFS protocol and it is very difficult to think that an "in vitro" model is able to perfectly re-create the physiological, natural equilibrium between striatal neurotransmitters and neuromodulators. As described above, DA [18], ACh [17] and endocannabinoids [29] but also nitric oxide, GABA, adenosine and several other molecules have been demonstrated to influence striatal synaptic plasticity and pharmacological studies often concentrated on only one neurotransmitter system, "extrapolating" it from the context and thus, looking at only one piece of the puzzle.

Moreover, since MSNs are embedded in a rich interneuronal network, it is difficult to determine if DA, ACh and the other neurotransmitters affect MSNs synaptic plasticity directly or indirectly through synaptically coupled neurons.

\subsection{Influence of membrane potential Up and Down states on MSNs synaptic plasticity}

Two other central factors in determining the final direction of the synaptic plastic change have been recently reviewed elsewhere [18] and are represented by the physiological status of the neuron and by the intensity of DAergic signaling at the time of the repeated synaptic activation.

With regard to the first point, it is conceivable that during a cortically driven Up state leading to membrane potential depolarization it could be easier to obtain the removal of the $\mathrm{Mg}^{2+}$-dependent block of NMDA receptors. NMDA receptor "deinactivation" might thereby facilitate intracellular calcium rise which, in turn, may trigger the $\mathrm{Ca}^{2+}$-dependent molecular mechanisms underlying LTP induction [18].

\subsection{The central role of dopamine}

As introduced above, DA is essential for both LTD and LTP induction at corticostriatal synapses.

More in particular, the strength of the DAergic input reaching the synapses during the induction protocol and the differential activation of D1 vs D2 DA receptors are also crucial factors in determining the final effect of the repeated synaptic activity and probably represent determining factors during reward-related learning $[18,59]$.

As described above, LTP induction at corticostriatal synapses seems to require D1 DA receptor stimulation, while LTD requires both D2 and D1 DA receptors activation [7,39].

Nevertheless, a part from their specific role during LTD and LTP induction, it is still a matter of debate what is the real, final effect of D1 and D2 DA receptor stimulation on MSNs although it is widely accepted that it deeply depends on the physiological status of the neuron at the moment of DA receptor stimulation [62].

Indeed, DA receptors stimulation has a different effect depending on the degree of membrane depolarization at which the receptor is activated. At more depolarized levels, closer to the Up state, the ion-channel phenotype of MSNs seems to change, and D1 and $\mathrm{D} 2$ receptors stimulation generate responses that are different from those obtained at more hyperpolarized membrane potential levels [62].

D1 receptors are known to be positively coupled to adenylyl cyclase, thus causing, when activated, an increase in cytosolic cAMP levels, the subsequent activation of protein kinase A and several downstream effects, such as an increase in surface expression of both AMPA and NMDA receptors and the enhancement of NMDA receptor mediated currents [62]. Moreover, trough a L-type $\mathrm{Ca}^{2+}$ channels-depending mechanism, D1-receptors stimulation is able to promote synaptically driven plateau potentials of MSNs, "generating" an Up state in corticostriatal slices [62].

On the other side, D2 receptors are negatively coupled to adenylyl cyclase and seem to act reducing neuronal excitability, neuronal response to glutamatergic inputs, and MSNs responsiveness at Up state membrane potentials [62].

The lack of homogeneity in DA receptors expression in MSNs further complicates this issue, since the prevailing hypothesis is that D1 and D2 DA receptors are "segregated" in only one of the two striatal projecting pathways with D1 receptors exciting MSNs of the "direct" striatonigral pathway and D2 receptors inhibiting MSNs of the "indirect" striatopallidal pathway [26].

The hypothesis of a segregation of D1 and D2 receptors in two different striatal populations of MSNs is partially in contrast with several experimental results suggesting that LTD is expressed by the large majority of striatal MSNs (rather than only by a half of them) [7] and that in BAC transgenic mice in which D1 and D2 receptors expressing MSNs are labeled with EGFP, D2 receptor antagonists block LTD induction in both types of MSNs, and not only in D2expressing MSNs [66].

There are several theories that can potentially explain the fact that LTD is expressed in both striatonigral and striatopalllidal neurons. The first looks at the interneurons as main actors. Indeed, the activation of D2 receptors expressed by cholinergic interneurons might be required to reduce $A C h$ release and subsequently lower M1 ACh muscarinic receptor tone and disinhibit Cav1.3 $\mathrm{Ca}^{2+}$ channels on D1-expressing striatonigral MSNs, an event that has been demonstrated to mediate LTD induction [66]. Conversely, as far as it concerns D2-expressing striatopallidal MSNs, the activation of D1 receptors should be required to enhance NO release from NOS + interneurons, which, in turn, participates in the induction phase of LTD through the cGMP pathway [14].

A different theory is that the D2 receptor-dependence of LTD in D1-expressing striatonigral MSNs may essentially represent a "spill-over" artifact caused by the induction protocol, potentially leading to the D2-dependent production and release of endocannabinoids from neighboring striatopallidal neurons.

\subsection{The importance of the in vivo vs. in vitro experimental preparation}

Up to 1997, the knowledge about activity-dependent plasticity at synapses between the cerebral cortex and the neostriatum came exclusively from in vitro experiments and, as described above, the general idea was that LTD was the physiological form of neuroplasticity at corticostriatal synapses while LTP was inducible only after the pharmacological manipulation of the slice medium.

In 1997 the first demonstration of an in vivo form of synaptic plasticity was provided by Charpier and Deniau [21]. Surprisingly, the authors found that in their in vivo preparation, when coupled with postsynaptic depolarization, the tetanus induced a LTP of corticostriatal excitatory transmission which was prevented by the intracellular injection of a $\mathrm{Ca}^{2+}$ chelator [21] suggesting that LTP was, to all extents, a physiological form of synaptic plasticity expressed by corticostriatal synapses and not only the expression of a putative pathological process over-activating NMDA glutamate receptors. 


\section{Short-term intrinsic plasticity in striatal neurons}

In addition to LTP and LTD, which are probably the two main known forms of synaptic plasticity, it is well accepted that the synaptic throughput can be also modulated by enduring changes in neuronal intrinsic excitability resulting from changes in voltagegated ion channels function $[3,25,73]$.

Indeed, neuronal excitability is regulated by the activity and distribution of ion channels in the plasma membrane and voltagegated ion channels activity plays a crucial role in coupling EPSPs to action potentials and thus in modulating neuronal output and whole circuit dynamic $[3,25]$. Certain learning tasks produce enduring changes in the intrinsic excitability of neurons by changing the function of voltage-gated ion channels which, in turn, either amplify or attenuate the amplitude of the EPSP $[3,25]$.

This information-storage mechanism, called "intrinsic plasticity", has several critical implications on the computational activity of neural circuits. First, intrinsic plasticity seems to act to maintain homeostasis. Indeed, manipulations reducing neuronal networks activation (such as the application of TTX) tend to produce changes increasing spike probability and duration [73]. Conversely, manipulations enhancing neuronal activation (e.g. raising external $\mathrm{K}^{+}$) seem to act decreasing spike probability and duration [73]. A second, important role of intrinsic neuronal plasticity is represented by its potential effects in mediating synaptic metaplasticity, which is a higher order form of synaptic plasticity expressed as a change in the ability to induce subsequent synaptic plasticity [73]. Finally, intrinsic plasticity could provide a widespread postsynaptic mechanism by which neurons optimize their input-output relationship during repeated afferent activity [47].

Unfortunately, the mechanisms of intrinsic plasticity following physiological activity are only beginning to be unravelled. In particular, because different neuronal subcompartments express characteristic combinations of ion-channel subunits, intrinsic plasticity mechanisms differ between neuronal dendrites, soma and axons [3].

Striatal MSNs exhibit specific electrical membrane properties that are known to influence their intrinsic excitability and their responsiveness to synaptic inputs. In particular, when MSNs membrane potential approaches the Down state the activation of a slowly inactivating $\mathrm{K}^{+}$current $\left(I_{\mathrm{As}}\right)$ is able to induce a slowing of the rate of depolarization which, in turn, cause a long latency of spike discharge [51].

In 2000, Mahon et al. have demonstrated that MSNs are able to optimize cortical information transfer by modifying their intrinsic excitability as a function of their past activation. In particular, the authors have shown that, if the intracellular injection of a test depolarizing current pulse was preceded by a $200 \mathrm{~ms}$ suprathreshold pulse, an increase in intrinsic excitability of the neuron was observed [44].

Pharmacological investigations and biophysical modeling have subsequently demonstrated that the observed decrease in spike threshold and the subsequent increase in firing probability seemed to depend on a time-dependent inactivation of the slowly inactivating $\mathrm{K}^{+}$current $I_{\text {As }}[45,46]$.

This form of short-term intrinsic excitability might be important in regulating the dynamics of basal ganglia circuit and the development of subsequent forms of long-term synaptic plasticity. Indeed, it establishes a short temporal window in which the striatal output neuron becomes more responsive to subsequent excitatory synaptic inputs. This latter event increases the probability that subsequent cortically evoked synaptic potentials will induce firing, providing a temporal link to optimize striatal cell firing during temporally ordered activity of converging cortical inputs [46].
Nevertheless, it is worth noting that many lines of evidence have led to the conclusion that it is not just the rate of spiking that encodes salient information in neuronal networks but, more critically, spike timing, which is the particular pattern in which spikes are evoked [73].

\section{Spike timing-dependent plasticity at corticostriatal synapses}

As introduced above, several studies suggest that the temporal relationship between activity in the pre- and postsynaptic elements constitutes a determinant factor for the induction of synaptic plasticity, influencing both the magnitude and the direction of the induced synaptic change [24].

On the basis of this hypothesis, synaptic strength can be affected in different ways according to whether a presynaptic spike closely precedes, or follows, an EPSP. In particular, in the majority of the systems in which the principle of spike timing-dependent plasticity (STDP) has been demonstrated, LTP is produced if the presynaptic signal precedes the postsynaptic response, while LTD is produced in the inverse scenario [24].

An hypothesis to explain the downstream effects of the temporal asymmetry in pre- vs post-synaptic spiking is that the relative timing of glutamate binding to NMDARs and the spiking of the postsynaptic dendrite influence the intracellular calcium levels required for either LTP or LTD [24]. Indeed, similar to LTP and LTD induced by conventional protocols, in STDP, pre-post spiking (EPSP followed by spike) leads to brief high-level $\mathrm{Ca}^{2+}$ influx due to effective activation of NMDARs (LTP pathway), while post-pre spiking (spike followed by EPSP) leads to a low-level $\mathrm{Ca}^{2+}$ rise (LTD pathway) [65].

At corticostriatal synapses, the idea of a critical role of the precise timing of spiking activity was proposed several years ago [7].

Fino et al. have investigated the role of the potential presence of an "STDP rule" at corticostriatal synapses [27]. For this purpose, they evoked a postsynaptic action potential by applying a supra threshold depolarizing pulse either before or after the stimulation of cortical afferences.

Surprisingly, the authors have found that the corticostriatal network expresses STDP forms that are reversed to those observed in other brain structures. Indeed, an LTP occurred when the postsynaptic action potential of the MSN neuron happened before a cortical stimulation, whereas an LTD was observed when a postsynaptic action potential was triggered in a MSN after cortical stimulation [27].

The presence of a reversed STDP rule at corticostriatal synapses is probably the consequence of several convergent factors that are known to be able to influence STDP orientation, such as (i) the nature of the neuron (excitatory vs inhibitory), (ii) the architecture of the dendritic tree and (iii) the resting membrane potential [27]. MSNs indeed exhibit a very hyperpolarized resting membrane potential, are GABAergic and have a highly branched spiny dendritic tree.

More recently, Pawlak and Kerr have also investigated the presence of STDP at corticostriatal synapses showing that timing of single postsynaptic action potentials (APs) relative to the cortically evoked EPSP determines both the direction and the strength of synaptic plasticity in MSNs [54]. In this study, the authors provide evidence that also striatal neurons follow the classical STDP rule. In particular, they have demonstrated that single APs occurring $30 \mathrm{~ms}$ before the cortically evoked EPSP induced LTD, whereas APs occurring $10 \mathrm{~ms}$ after the EPSP induced LTP and that DA D1/D5 receptor activation is critically required for striatal STDP [54]. 


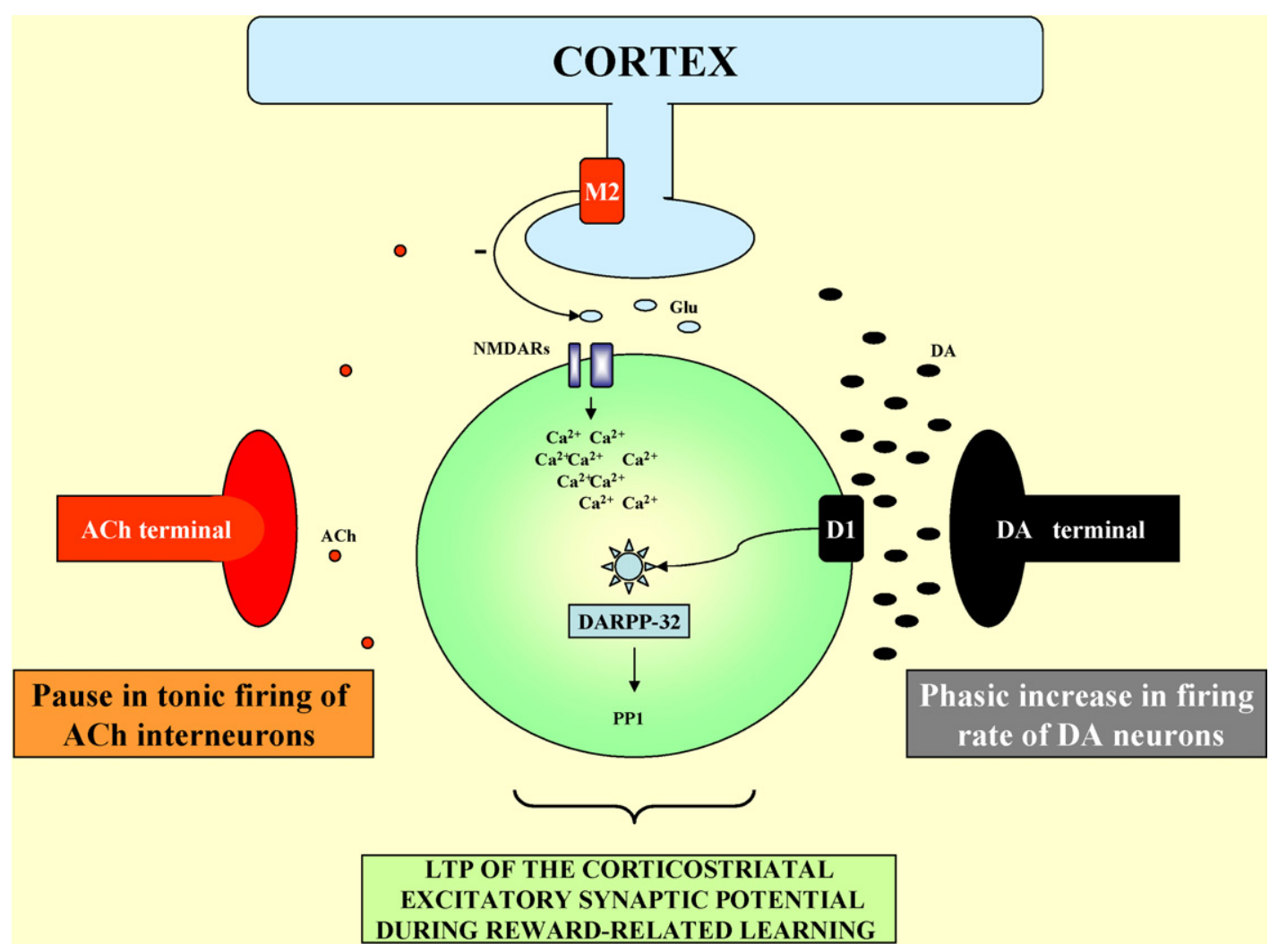

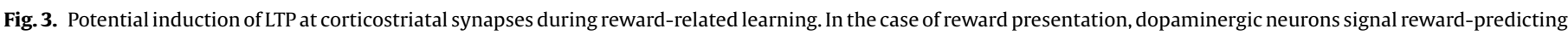

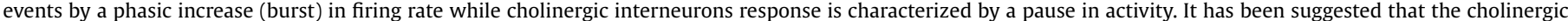

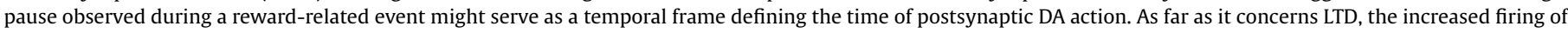

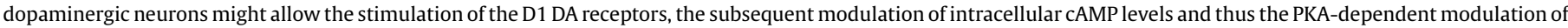

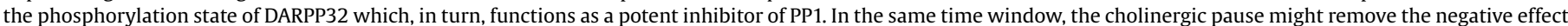

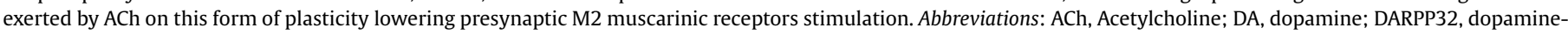
and cAMP-regulated phosphoprotein $32 \mathrm{kDa}$; Glu, glutamate; PP1, protein phosphatase 1.

Nevertheless, apart from the cause underlying the possible presence of a reversed STDP rule at corticostriatal synapses onto MSNs, it is worth to note that the expression of this form of plasticity might have several computational implications. In particular, STDP might underlie the bidirectionality of synaptic modifications (LTD and LTP), helping the neural circuit to maintain its net synaptic excitation at a stable level and to regulate the temporal dynamics of the circuit [24].

\section{The pursuit of happiness: reward-related learning and corticostriatal synaptic plasticity}

What is the force pushing human actions? A sole answer to this question probably does not exist and the issue of understanding human behavior seems to be complicated, rather than simplified, by the merging of philosophical, religious and neurobiological considerations.

It is widely accepted that animals, including humans, shape their behavior on the basis of experience and that motivation is characterized by action, either to increase the probability of an outcome (appetitive motivation), or to reduce it (aversive motivation) [61].

Learned responses require knowledge about the relationships between stimuli and actions and they seem to be essential for reward prediction and for driving goal-directed actions [5]. The natural incentives that shape behavior reach the central circuitry of motivation trans-synaptically, via the five senses [71] and are probably able to induce, via the modulation of neuronal activity, the long-lasting modifications of the efficacy of synaptic transmission that are required to learn about relationships among stimuli and consequences of actions.

In particular, it is conceivable that in the functional circuit underlying reward-related learning the induction of long-lasting synaptic memories underlie positive reinforcement and habit formation during physiological conditions and addictive and compulsive behaviors during pathological situations.

As introduced above, the striatum is thought to be composed by two main substructures the "dorsal" and the "ventral" striatum or nucleus accumbens (NAc). These two regions are very similar but seem to differ with regard to afferent and efferent circuitry and specific physiological role. Indeed, the dorsal striatum (in particular its medial and lateral regions) is preferentially innervated by associative and sensorimotor areas of the cortex, receives dopaminergic inputs from the SNpc and seems to mainly participate to movement generation and learning. Conversely, the NAc primarily receives afferents from limbic structures, receives dopaminergic afferents from the ventral tegmental area (VTA) and constitutes a primary component of the "mesolimbic pathway" playing a crucial role during reward. The central role played by striatal LTP and LTD during motor learning and generation have been extensively reviewed elsewhere as well as the dramatic consequences of striatal dopaminergic denervation on both motor ability and synaptic plasticity induction $[11,15,18,56]$. Conversely, the mechanisms underlying the potential association between striatal neuroplasticity and reward-related behaviors and learning are still far from being elucidated. 


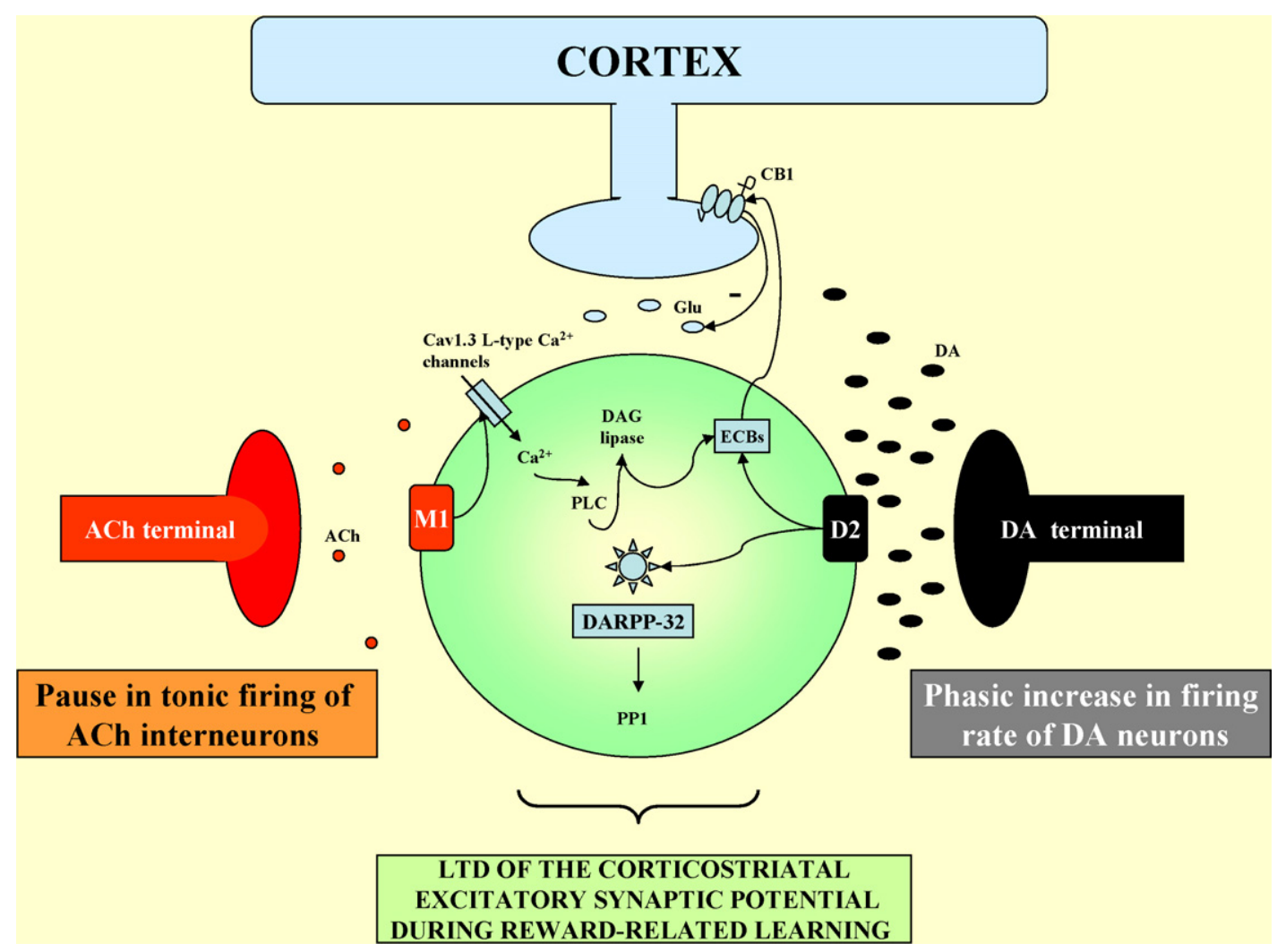

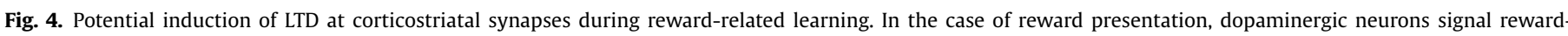

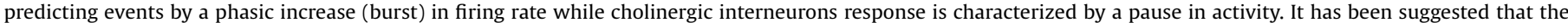

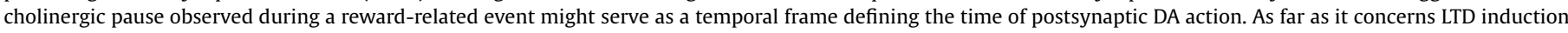

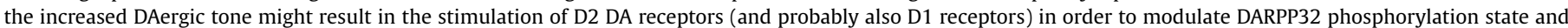

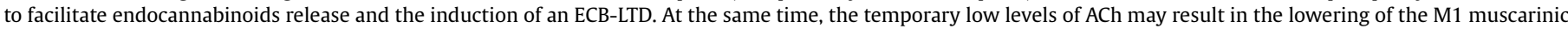

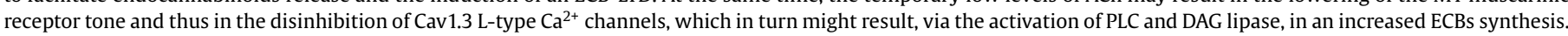

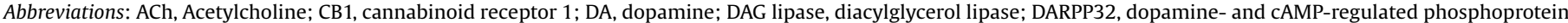
$32 \mathrm{kDa}$; ECBs, endocannabinoids, Glu, glutamate; PLC, phospholipase C; PP1, protein phosphatase 1.

The striatum might play a crucial role in reward-related memory. In particular, a cooperative role should be played by DAergic and cholinergic inputs converging on striatal MSNs during precise time windows.

Mesostriatal DAergic neurons (DANs) and striatal cholinergic interneurons seem to be pivotal for signaling unexpected primary rewards in addition to the learning and signaling of environmental cues the predict reward [23].

Interestingly, although both these two classes of neurons are somehow activated during reward-related events, their responses differ. In particular, in the case of reward presentation, DANs signal reward-predicting events by a phasic increase (burst) in firing rate, while cholinergic interneurons response is characterized by a pause in activity (a pause that sometimes occurs within a possible triphasic template, comprising pre-excitation, pause, and post-excitation phases) [23].

Which might be the potential usefulness of this AChergicDAergic neurons time locking during reward-related events? More in particular, might it represent an event required to obtain a determined synaptic concentration of both ACh and DA able to modulate the induction of synaptic long-lasting changes?

It has been suggested that the cholinergic pause observed during a reward-related event might serve as a temporal frame defining the time over which the information content of the DA signal will be processed postsynaptically [50,23].

According to the current knowledge about the relative roles played by DA and ACh during corticostriatal synaptic plasticity it is possible to hypothesize that reward-related learning at corticostri- atal synapses may depend on this time-locked opposite responses of DANs and cholinergic interneurons.

Indeed, the contemporaneous presence, during a rewardrelated event of high levels of synaptic DA and low levels of synaptic ACh might potentially facilitate both LTD and LTP induction (Figs. 3 and 4).

As far as it concerns LTP, the increased firing of DANs might allow the stimulation of the D1 DA receptors [39], while the cholinergic pause might remove the negative effect exerted by ACh on this form of plasticity lowering M2 muscarinic receptors stimulation [12] (Fig. 3).

Conversely, with regard to LTD, the increased DAergic tone may be required to stimulate D2 DA receptors (and probably also D1 receptors) [7] while the temporary low levels of ACh may result in the lowering of the M1 muscarinic receptor tone and thus in the disinhibition of Cav1.3 $\mathrm{Ca}^{2+}$ channels [66] (Fig. 4).

The proposed model is voluntarily over-simplified and a number of factors may potentially bias the synapse toward the preferential induction of an LTP and or LTD including the large majority of those described above. Nevertheless, it provides a working hypothesis showing that either LTP or LTD might be potentially triggered during reward-related learning at corticostriatal synapses.

The central effect played by DA in this scenario is consistent with the acknowledged roles of this neurotransmitter in motivation and reward [59].

Accordingly, neuroleptic drugs (DA antagonists) seem to attenuate the motivation to act before they compromise the ability (intended as motor ability) to act [71]. 
The suggestion that the mechanisms underlying drug addiction might involve natural reward systems and striatal synaptic plasticity has also stimulated interest [30].

Indeed, major drugs of abuse (including heroin and other opiates, cocaine, amphetamine, and nicotine) lead to increases in DA concentration in the ventral striatum and frontal cortex, which appears to be a crucial mechanism of drug addiction [59] and are both rewarding (interpreted by the brain as intrinsically positive) and reinforcing (behaviors associated with such drugs tend to be repeated) [37].

Thus, it is conceivable that the powerful behavioral control exerted by addictive drugs might result from the brain's inability to distinguish between the activation of reward circuitry by naturally rewarding activities, such as eating, and by the consumption of drugs. More in particular, the increase in synaptic DA induced by drugs might mimic the physiological condition observed during reward-related learning and thus induce longlasting changes in the efficacy of synaptic transmission finally resulting in drug-seeking despite the efforts to abstain. Accordingly, evidence suggests that the main forms of striatal synaptic plasticity might be profoundly altered after the repeated administration of addictive drugs $[20,64,2,36]$. An extensive description of the complex effects exerted by drugs of abuse of striatal function and plasticity would be beyond the scope of the article. Nevertheless, it is worth citing that, according to the hypothesis of a role of synaptic changes in mediating the behavioral aspects of the advanced stages of addiction, abnormalities in striatal synaptic plasticity have been demonstrated to occur after the administration of different drugs.

Evidence exists that, after repeated exposure to cocaine, the ratio of AMPA to NMDA receptor-mediated excitatory postsynaptic currents (EPSCs) is decreased at synapses made by prefrontal cortical afferents onto MSNs in the shell of the NAc and that the amplitude of LTD at these synapses is also decreased [64]. It has been also demonstrated that the chronic, but not the acute, administration of cocaine is able to block the reversal of LTP at corticostriatal synapses [20].

The exposure to another drug, the dopamine-releaser methamphetamine, is able to elicit a long-lasting presynaptic depression at corticostriatal terminals that is reversed by the methamphetamine readministration [2].

Studies investigating the long-term synaptic impact of cannabinoid exposure have also been carried out and have shown that, in the NAc of rats chronically treated with Delta9-THC, LTD is impaired and the sensitivity of GABAergic and glutamatergic synapses to both cannabinoids and opioids is reduced [36].

\section{Conclusions and future perspectives}

In the last years, significant progresses have been made in the specific field of synaptic plasticity at corticostriatal synapses and some of the molecular mechanisms underlying the induction of both striatal LTP and LTD have been partially elucidated.

Hopefully, in the future, a more profound knowledge of the mechanisms underlying these synaptic memory processes will allow to design pharmacological compounds able to directly target selective corticostriatal neuronal connections (e.g. onto MSNs, GABAergic or cholinergic interneurons) and to bias the synapse toward the preferential induction of a previously selected form of synaptic plasticity.

\section{Acknowledgements}

This work was supported by the European Community (HEALTH-2007-22918, REPLACES) (P.C.), Progetti Finalizzati Min- istero della Salute 2006-2008 (P.C., B.P.), Progetto Strategico 2008 (P.C., B.P.), Fondazione Cassa di Risparmio di Perugia (P.C.) and by the EU Grants cNEUPRO (contract No. LSHM-CT-2007-037950) (L.P.). V.B. is a recipient of a PhD student bursary from the University of Calabria, PhD course on Pharmacology and Biochemistry of Cell Death, Rende (CS), Italy.

\section{References}

[1] Abbott LF, Nelson SB. Synaptic plasticity: taming the beast. Nat Neurosci 2000;3(Suppl.):1178-83.

[2] Bamford NS, Zhang H, Joyce JA, Scarlis CA, Hanan W, Wu NP, et al. Repeated exposure to methamphetamine causes long-lasting presynaptic corticostriatal depression that is renormalized with drug readministration. Neuron 2008;58(1):89-103.

[3] Beck H, Yaari Y. Plasticity of intrinsic neuronal properties in CNS disorders. Nat Rev Neurosci 2008;9(5):357-69.

[4] Berretta N, Nisticò R, Bernardi G, Mercuri NB. Synaptic plasticity in the basal ganglia: a similar code for physiological and pathological conditions. Prog Neurobiol 2008;84(4):343-62.

[5] Berridge KC, Robinson TE. Parsing reward. Trends Neurosci 2003;26(9):50713.

[6] Bracci E, Centonze D, Bernardi G, Calabresi P. Dopamine excites fast-spiking interneurons in the striatum. J Neurophysiol 2002;87(4):2190-4.

[7] Calabresi P, Maj R, Pisani A, Mercuri NB, Bernardi G. Long-term synaptic depression in the striatum: physiological and pharmacological characterization. J Neurosci 1992;12(11):4224-33.

[8] Calabresi P, Pisani A, Mercuri NB, Bernardi G. Long-term potentiation in the striatum is unmasked by removing the voltage-dependent magnesium block of NMDA receptor channels. Eur J Neurosci 1992;4(10):929-35.

[9] Calabresi P, Mercuri NB, Sancesario G, Bernardi G. Electrophysiology of dopamine-denervated striatal neurons. Implications for Parkinson's disease. Brain 1993;116(2):433-52.

[10] Calabresi P, Pisani A, Mercuri NB, Bernardi G. Post-receptor mechanisms underlying striatal long-term depression. J Neurosci 1994;14:4871-81.

[11] Calabresi P, Pisani A, Mercuri NB, Bernardi G. The corticostriatal projection: from synaptic plasticity to dysfunctions of the basal ganglia. Trends Neurosci 1996;19(1):19-24.

[12] Calabresi P, Centonze D, Gubellini P, Pisani A, Bernardi G. Blockade of M2like muscarinic receptors enhances long-term potentiation at corticostriatal synapses. Eur J Neurosci 1998;10(9):3020-3.

[13] Calabresi P, Centonze D, Gubellini P, Bernardi G. Activation of M1-like muscarinic receptors is required for the induction of corticostriatal LTP. Neuropharmacology 1999;38(2):323-6.

[14] Calabresi P, Gubellini P, Centonze D, Sancesario G, Morello M, Giorgi M, et al. A critical role of the nitric oxide/cGMP pathway in corticostriatal long-term depression. J Neurosci 1999;19(7):2489-99.

[15] Calabresi P, Giacomini P, Centonze D, Bernardi G. Levodopa-induced dyskinesia: a pathological form of striatal synaptic plasticity? Ann Neurol 2000;47(4 Suppl. 1):S60-8.

[16] Calabresi P, Saulle E, Centonze D, Pisani A, Marfia GA, Bernardi G. Post-ischaemic long-term synaptic potentiation in the striatum: a putative mechanism for cell type-specific vulnerability. Brain 2002;125(Pt 4):844-60.

[17] Calabresi P, Picconi B, Parnetti L, Di Filippo M. A convergent model for cognitive dysfunctions in Parkinson's disease: the critical dopamine-acetylcholine synaptic balance. Lancet Neurol 2006;5(11):974-83.

[18] Calabresi P, Picconi B, Tozzi A, Di Filippo M. Dopamine-mediated regulation of corticostriatal synaptic plasticity. Trends Neurosci 2007;30(5):211-9.

[19] Centonze D, Gubellini P, Picconi B, Calabresi P, Giacomini P, Bernardi G. Unilateral dopamine denervation blocks corticostriatal LTP. J Neurophysiol 1999;82(6):3575-9.

[20] Centonze D, Costa C, Rossi S, Prosperetti C, Pisani A, Usiello A, et al. Chronic cocaine prevents depotentiation at corticostriatal synapses. Biol Psychiatry 2006;60(5):436-43.

[21] Charpier S, Deniau JM. In vivo activity-dependent plasticity at cortico-striatal connections: evidence for physiological long-term potentiation. Proc Natl Acad Sci USA 1997;94(13):7036-40.

[22] Citri A, Malenka RC. Synaptic plasticity: multiple forms, functions, and mechanisms. Neuropsychopharmacology 2008;33(1):18-41.

[23] Cragg SJ. Meaningful silences: how dopamine listens to the ACh pause. Trends Neurosci 2006;29(3):125-31.

[24] Dan Y, Poo MM. Spike timing-dependent plasticity of neural circuits. Neuron 2004;44(1):23-30.

[25] Daoudal G, Debanne D. Long-term plasticity of intrinsic excitability: learning rules and mechanisms. Learn Mem 2003;10(6):456-65.

[26] DeLong MR, Wichmann T. Circuits and circuit disorders of the basal ganglia. Arch Neurol 2007;64(1):20-4.

[27] Fino E, Glowinski J, Venance L. Bidirectional activity-dependent plasticity at corticostriatal synapses. J Neurosci 2005;25(49):11279-87.

[28] Fino E, Deniau JM, Venance L. Cell-specific spike-timing-dependent plasticity in GABAergic and cholinergic interneurons in corticostriatal rat brain slices. J Physiol 2008;586(1):265-82. 
[29] Gerdeman GL, Ronesi J, Lovinger DM. Postsynaptic endocannabinoid release is critical to long-term depression in the striatum. Nat Neurosci 2002; 5(5):446-51.

[30] Gerdeman GL, Partridge JG, Lupica CR, Lovinger DM. It could be habit forming: drugs of abuse and striatal synaptic plasticity. Trends Neurosci 2003;26(4):184-92.

[31] Giuffrida A, Parsons LH, Kerr TM, Rodríguez de Fonseca F, Navarro M, Piomelli D. Dopamine activation of endogenous cannabinoid signaling in dorsal striatum. Nat Neurosci 1999;2(4):358-63.

[32] Grillner S, Hellgren J, Ménard A, Saitoh K, Wikström MA. Mechanisms for selection of basic motor programs-roles for the striatum and pallidum. Trends Neurosci 2005;28(7):364-70.

[33] Gubellini P, Saulle E, Centonze D, Bonsi P, Pisani A, Bernardi G, et al. Selective involvement of mGlu1 receptors in corticostriatal LTD. Neuropharmacology 2001;40(7):839-46.

[34] Gubellini P, Pisani A, Centonze D, Bernardi G, Calabresi P. Metabotropic glutamate receptors and striatal synaptic plasticity: implications for neurological diseases. Prog Neurobiol 2004;74(5):271-300.

[35] Hikosaka O, Nakahara H, Rand MK, Sakai K, Lu X, Nakamura K, et al. Parallel neural networks for learning sequential procedures. Trends Neurosci 1999;22(10):464-71.

[36] Hoffman AF, Oz M, Caulder T, Lupica CR. Functional tolerance and blockade of long-term depression at synapses in the nucleus accumbens after chronic cannabinoid exposure. J Neurosci 2003;23(12):4815-20.

[37] Hyman SE, Malenka RC. Addiction and the brain: the neurobiology of compulsion and its persistence. Nat Rev Neurosci 2001;2(10):695-703.

[38] Kawaguchi Y, Wilson CJ, Augood SJ, Emson PC. Striatal interneurones: chemical, physiological and morphological characterization. Trends Neurosci 1995;18(12):527-35.

[39] Kerr JN, Wickens JR. Dopamine D-1/D-5 receptor activation is required for long-term potentiation in the rat neostriatum in vitro. J Neurophysiol 2001;85(1):117-24.

[40] Kreitzer AC, Malenka RC. Dopamine modulation of state-dependent endocannabinoid release and long-term depression in the striatum. J Neurosci 2005;25(45):10537-45.

[41] Kreitzer AC, Malenka RC. Endocannabinoid-mediated rescue of striatal LTD and motor deficits in Parkinson's disease models. Nature 2007;445(7128):643-7.

[42] Lang AE, Lozano AM. Parkinson's disease. First of two parts. N Engl J Med 1998;339(15):1044-53.

[43] Lang AE, Lozano AM. Parkinson's disease. Second of two parts. N Engl J Med 1998;339(16):1130-43.

[44] Mahon S, Delord B, Deniau JM, Charpier S. Intrinsic properties of rat striatal output neurones and time-dependent facilitation of cortical inputs in vivo. J Physiol 2000;527(Pt 2):345-54

[45] Mahon S, Deniau JM, Charpier S, Delord B. Role of a striatal slowly inactivating potassium current in short-term facilitation of corticostriatal inputs: a computer simulation study. Learn Mem 2000;7(5):357-62.

[46] Mahon S, Casassus G, Mulle C, Charpier S. Spike-dependent intrinsic plasticity increases firing probability in rat striatal neurons in vivo. J Physiol 2003;550(Pt 3):947-59.

[47] Mahon S, Deniau JM, Charpier S. Corticostriatal plasticity: life after the depression. Trends Neurosci 2004;27(8):460-7.

[48] Malenka RC, Nicoll RA. Long-term potentiation-a decade of progress? Science 1999;285(5435):1870-4.

[49] Massey PV, Bashir ZI. Long-term depression: multiple forms and implications for brain function. Trends Neurosci 2007;30(4):176-84.

[50] Morris G, Arkadir D, Nevet A, Vaadia E, Bergman H. Coincident but distinct messages of midbrain dopamine and striatal tonically active neurons. Neuron 2004;43(1):133-43.
[51] Nisenbaum ES, Xu ZC, Wilson CJ. Contribution of a slowly inactivating potassium current to the transition to firing of neostriatal spiny projection neurons. J Neurophysiol 1994;71(3):1174-89.

[52] O'Dell TJ, Kandel ER. Low-frequency stimulation erases LTP through an NMDA receptor-mediated activation of protein phosphatases. Learn Mem 1994; $1: 129-39$.

[53] Partridge JG, Tang KC, Lovinger DM. Regional and postnatal heterogeneity of activity-dependent long-term changes in synaptic efficacy in the dorsal striatum. J Neurophysiol 2000;84(3):1422-9.

[54] Pawlak V, Kerr JN. Dopamine receptor activation is required for corticostriata spike-timing-dependent plasticity. J Neurosci 2008;28(10):2435-46.

[55] Picconi B, Centonze D, Håkansson K, Bernardi G, Greengard P, Fisone G, et al. Loss of bidirectional striatal synaptic plasticity in L-DOPA-induced dyskinesia. Nat Neurosci 2003;6(5):501-6.

[56] Pisani A, Centonze D, Bernardi G, Calabresi P. Striatal synaptic plastic ity: implications for motor learning and Parkinson's disease. Mov Disord 2005;20(4):395-402.

[57] Pisani A, Bernardi G, Ding J, Surmeier DJ. Re-emergence of striatal cholinergic interneurons in movement disorders. Trends Neurosci 2007;30(10):545-53.

[58] Schotanus SM, Chergui K. Dopamine D1 receptors and group I metabotropic glutamate receptors contribute to the induction of long-term potentiation in the nucleus accumbens. Neuropharmacology 2008;54(5):837-44.

[59] Schultz W. Getting formal with dopamine and reward. Neuron 2002;36(2):241-63.

[60] Schultz W, Tremblay L, Hollerman JR. Changes in behavior-related neurona activity in the striatum during learning. Trends Neurosci 2003;26(6):321-8.

[61] Seymour B, Singer T, Dolan R. The neurobiology of punishment. Nat Rev Neurosci 2007;8(4):300-11.

[62] Surmeier DJ, Ding J, Day M, Wang Z, Shen W. D1 and D2 dopamine-receptor modulation of striatal glutamatergic signaling in striatal medium spiny neurons. Trends Neurosci 2007;30(5):228-35.

[63] Suzuki T, Miura M, Nishimura K, Aosaki T. Dopamine-dependent synaptic plasticity in the striatal cholinergic interneurons. J Neurosci 2001;21(17):6492-501.

[64] Thomas MJ, Beurrier C, Bonci A, Malenka RC. Long-term depression in the nucleus accumbens: a neural correlate of behavioural sensitization to cocaine. Nat Neurosci 2001;4(12):1217-23.

[65] Tzounopoulos T, Rubio ME, Keen JE, Trussell LO. Coactivation of pre- and postsynaptic signaling mechanisms determines cell-specific spike-timing-dependent plasticity. Neuron 2007;54(2):291-301.

[66] Wang Z, Kai L, Day M, Ronesi J, Yin HH, Ding J, et al. Dopaminergic control of corticostriatal long-term synaptic depression in medium spiny neurons is mediated by cholinergic interneurons. Neuron 2006;50(3):443-52.

[67] Voorn P, Vanderschuren LJ, Groenewegen HJ, Robbins TW, Pennartz CM. Putting a spin on the dorsal-ventral divide of the striatum. Trends Neurosci 2004;27(8):468-74.

[68] Wickens JR, Begg AJ, Arbuthnott GW. Dopamine reverses the depression of rat corticostriatal synapses which normally follows high-frequency stimulation of cortex in vitro. Neuroscience 1996;70(1):1-5.

[69] Wilson CJ, Kawaguchi Y. The origins of two-state spontaneous membrane potential fluctuations of neostriatal spiny neurons. J Neurosci 1996;16(7):2397-410.

[70] Wilson RI, Nicoll RA. Endocannabinoid signaling in the brain. Science 2002;296(5568):678-82.

[71] Wise RA. Dopamine, learning and motivation. Nat Rev Neurosci 2004 5(6):483-94.

[72] Yin HH, Knowlton BJ. The role of the basal ganglia in habit formation. Nat Rev Neurosci 2006;7(6):464-76.

[73] Zhang W, Linden DJ. The other side of the engram: experience-driven changes in neuronal intrinsic excitability. Nat Rev Neurosci 2003;4(11):885-900. 